OPEN ACCESS

Edited by:

Ilias Travlos,

Agricultural University of Athens,

Greece

Reviewed by:

Thomas Debener.

Leibniz University of Hanover,

Germany

Paul John Hunter,

University of Warwick, UK

*Correspondence:

Todd A. Gaines

todd.gaines@colostate.edu

Specialty section:

This article was submitted to Agroecology and Land Use Systems,

a section of the journal

Frontiers in Plant Science

Received: 14 February 2017 Accepted: 21 April 2017

Published: 08 May 2017

Citation:

Patterson EL, Fleming MB, Kessler KC, Nissen SJ and

Gaines TA (2017) A KASP Genotyping Method to Identify Northern Watermilfoil, Eurasian

Watermilfoil, and Their Interspecific Hybrids. Front. Plant Sci. 8:752. doi: 10.3389/fpls.2017.00752

\title{
A KASP Genotyping Method to Identify Northern Watermilfoil, Eurasian Watermilfoil, and Their Interspecific Hybrids
}

Eric L. Patterson, Margaret B. Fleming, Kallie C. Kessler, Scott J. Nissen and Todd A. Gaines*

Department of Bioagricultural Sciences and Pest Management, Colorado State University, Fort Collins, CO, USA

The invasive aquatic plant Eurasian watermilfoil (Myriophyllum spicatum L.) can hybridize with the related North American native species northern watermilfoil (M. sibiricum Kom.). Hybrid watermilfoil (M. spicatum $\times M$. sibiricum) populations have higher fitness and reduced sensitivity to some commonly used aquatic herbicides, making management more difficult. There is growing concern that management practices using herbicides in lakes with mixed populations of watermilfoil species may further select for hybrid individuals due to the difference in herbicide sensitivity. Accurate and cost-effective identification of rare hybrid individuals within populations is therefore critical for herbicide management decisions. Here we describe KASP assays for three SNPs in the ITS region to genotype individuals from both parental watermilfoil species and their hybrid, using synthesized plasmids containing the respective sequences as positive controls. Using KASP we genotyped 16 individuals from one lake and 23 individuals from a second lake, giving a highly accurate picture of Myriophyllum species distribution dynamics. We identified one hybrid individual among 16 samples from one lake, a discovery rate of $<10 \%$. Discriminant analysis showed that while a single SNP was generally sufficient for genotyping an individual, using multiple SNPs increased the reliability of genotyping. In the future, the ability to genotype many samples will provide the ability to identify the presence of rare individuals, such as a less common parental species or the inter-specific hybrid. Lakes with complex species distribution dynamics, such as a low proportion of hybrids, are where herbicide application must be carefully chosen so as not to select for the more vigorous and less herbicide-sensitive hybrid individuals.

Keywords: genotyping, KASP, invasive, aquatic weed, hybridization

\section{INTRODUCTION}

The invasive aquatic plant Eurasian watermilfoil (Myriophyllum spicatum L.) was introduced to the United States from Asia during the 1940s (Couch and Nelson, 1988; Moody et al., 2016). After introduction, this submersed species spread rapidly throughout the USA, forming dense monotypic mats that have caused economic and ecological damage to infested lakes, streams, and reservoirs (Eiswerth et al., 2000; Olden and Tamayo, 2014). The decrease in native plant diversity that occurs after M. spicatum invasion is an alarming ecological impact (Madsen et al., 1991). 
Like many aquatic plants, $M$. spicatum is a perennial that often reproduces asexually, a strategy that has the advantage of cloning better-adapted genotypes in stable environments (Philbrick and Les, 1996). M. spicatum uses a very simple form of asexual reproduction called autofragmentation. Autofragmentation usually occurs soon after flowering when stems that are relatively fragile break off from the parent plant and float away as structures that can start new colonies (Grace, 1993). One characteristic of these shoot pieces that adds to the success of autofragmentation is the development of adventitious roots. M. spicatum has retained the ability to reproduce sexually using a common strategy of many aquatic angiosperms. Modified stems project from the vegetative mat and terminate in monecious flowers above the water surface. This allows for flowers to be wind-pollinated. Sexual reproduction and subsequent seed production are often overlooked as an important survival strategy for M. spicatum. Seed production can be an insurance against local extinction because there is some seed dormancy (Grace and Wetzel, 1978). Sexual reproduction can also produce new genotypes that could be better adapted to changing environments.

Environmental change for aquatic plants is usually thought of in terms of salinity, $\mathrm{pH}$, or turbidity, but not necessarily annual applications of aquatic herbicides. It is now apparent that M. spicatum has hybridized with the related North American species northern watermilfoil (M. sibiricum Kom.) (Moody and Les, 2007; Zuellig and Thum, 2012; Grafe et al., 2015). In

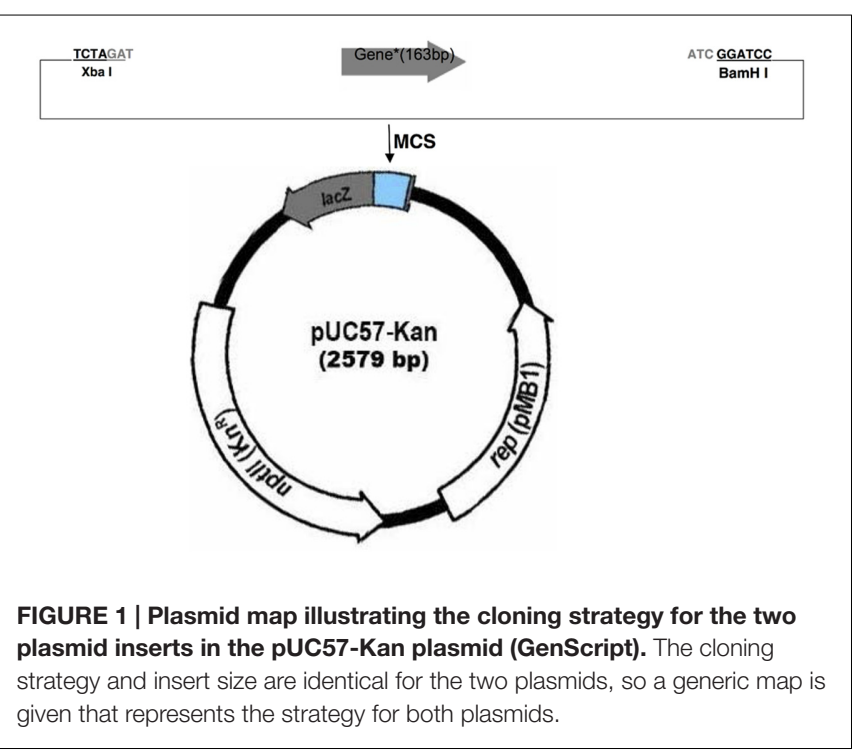

the absence of herbicide selection pressure, hybrid watermilfoil (M. spicatum $\times$ M. sibiricum) populations appear to have higher fitness, manifested as a faster and more aggressive growth rate than either parental species in both laboratory and field conditions (LaRue et al., 2013; Hovick and Whitney, 2014). Some hybrid populations are also less sensitive to several commonly used aquatic herbicides, including 2,4-D, fluridone, norflurazon, and topramazone (LaRue et al., 2013; Berger et al., 2015). There is growing concern that current management practices in lakes with mixed populations of watermilfoil species, which rely heavily on herbicide application, may further select for hybrid populations due to the difference in herbicide sensitivity.

Several methods to accurately identify $M$. spicatum, M. sibiricum, and M. spicatum $\times$ M. sibiricum hybrid individuals using morphological characteristics have been proposed. Morphological characteristics, while sufficient to distinguish between $M$. spicatum and $M$. sibiricum, are no longer reliable once hybrid individuals are present, as the hybrid characteristics are often intermediate between the two species (e.g., the number of pinnae or leaflet pairs) (Coffey and McNabb, 1974; Moody and Les, 2007).

Sufficient genetic variation exists between the two species that genotyping is an accurate method for species identification (Moody and Les, 2002; Sturtevant et al., 2009). Current methods rely on 23 intra-genic polymorphic single nucleotide polymorphisms (SNPs) within the first and second nuclear ribosomal internal transcribed spacer regions (ITS1 and ITS2) of M. spicatum and M. sibiricum (Moody and Les, 2002). Of these SNPs, 11 clearly distinguish between M. spicatum and $M$. sibiricum. When a single individual is heterozygous for both alleles of a single SNP, it indicates the individual is an interspecific hybrid. A hybrid individual will also be heterozygous for the remaining 10 SNPs due to linkage of the SNPs within the ITS regions.

Single nucleotide polymorphism genotyping in these species has been performed using several methods. Originally, the ITS region was amplified via polymerase chain reaction (PCR), the PCR products were cloned, and multiple clones were sequenced to determine their genotype (Grafe et al., 2015). This process requires the longest time and highest cost per sample of available methods. Subsequently, genotyping was streamlined with the development of a PCR restriction fragment length polymorphism (PCR-RFLP) assay using either a BmtI or FspI restriction digest that cut at base pair (bp) 274 or 551 of the ITS amplicon, respectively. By eliminating the cloning and sequencing for species identification with the PCR-RFLP assay, Grafe et al. (2015) were able to substantially decrease the amount of time

TABLE 1 | The full sequence of the plasmid inserts for $M$. sibiricum and $M$. spicatum positive plasmid controls.

\begin{tabular}{|c|c|c|c|c|}
\hline Plasmid & Gene name & Length (bp) & Vector name & Sequence $\left(5^{\prime}-3^{\prime}\right)$ \\
\hline 2 & M_Spi_Positive_Control & 163 & pUC57-Kan & $\begin{array}{l}\text { CATGACGAACTTAGCACACCACTAGCCGACTTGTGCGGCAGCGGCGTTGCAAACTTCGATACCTA } \\
\text { CAAAGCCCACCCTTCAAGGATAAGGCGCTGCGGAAGCAGATATTGGATAACTCAGCCTTGTTGCG } \\
\text { CCGTGCCCGCCGTGCCCCTTGGAGCTCAGCAT }\end{array}$ \\
\hline
\end{tabular}


and money per sample required for positive species identification of individual watermilfoil specimens. The higher throughput enabled larger sample sizes, providing a more accurate estimate of Myriophyllum species distribution dynamics.

Recent advances in SNP genotyping provide more costeffective and accurate results than PCR-RFLP. Currently, the Kompetitive Allele Specific PCR (KASP) assay is a common technique for genotyping SNPs. This assay is used in several fields of study, including plant breeding, disease identification, and species identification (Semagn et al., 2014). KASP is able to discriminate between two alleles of a SNP using a common reverse primer paired with two forward primers, one specific to each allele. Each forward primer also has a nucleotide sequence that hybridizes to either the HEX or FAM fluorophore quencher. Amplification proceeds using stringent conditions to permit forward primers to bind only if they are perfectly complementary to the template sequence. Fluorophores are released from the quencher molecule when a forward primer is incorporated in a PCR product, causing the released fluorophore to fluoresce. This fluorescence is detected at the end of the assay using a real-time PCR machine, and the proportion of fluorescence from HEX, FAM, or both indicates the genotype of the sample.

Kompetitive Allele Specific PCR genotyping has several advantages compared to other genotyping assays. KASP assays are more convenient, as they are both faster and less expensive. Eighty or more individuals can be genotyped simultaneously on a standard 96-well plate, giving a much more accurate view of the Myriophyllum species distribution dynamics, and increasing the likelihood of detecting a rare hybrid individual. KASP assay design is very flexible, as useful SNPs are not limited to available restriction enzyme recognition sites, and primers can even cover stretches of sequence containing multiple SNPs by incorporating degenerate or mixed bases into the primer sequence. KASP assays are quantitative and therefore amenable to statistical analysis, such as assigning probabilities to genotyping calls. Data from

TABLE 2 | Kompetitive Allele Specific PCR primers for SNPs 118, 363, and 478 in the Myriophyllum ITS region.

\begin{tabular}{|c|c|c|}
\hline Primer name & Primer sequence $\left(5^{\prime}-3^{\prime}\right)$ & $\begin{array}{r}\text { OligoAnalyzer } \\
\text { predicted melti } \\
\text { temperature }\end{array}$ \\
\hline \multicolumn{3}{|l|}{ SNP 118 (G/A) } \\
\hline M. sibiricum FP-118 & CATGACGWACTTAGCACACCG & $55.9^{\circ} \mathrm{C}$ \\
\hline M. spicatum FP-118 & CATGACGAACTTAGCACACCA & $55.2^{\circ} \mathrm{C}$ \\
\hline Universal RP-118 & TAGGTATCGAAGTITGCAACGC & $55.5^{\circ} \mathrm{C}$ \\
\hline \multicolumn{3}{|l|}{ SNP 363 (A/G) } \\
\hline M. sibiricum FP-363 & CAATATCTGCTTCCGCAGCA & $55.6^{\circ} \mathrm{C}$ \\
\hline M. spicatum FP-363 & CAATATCTGCTTCCGCAGCG & $56.6^{\circ} \mathrm{C}$ \\
\hline Universal RP-363 & CAAAGCCCACCCTTCAAGGA & $57.7^{\circ} \mathrm{C}$ \\
\hline \multicolumn{3}{|l|}{ SNP $478(T / C)$} \\
\hline M. sibiricum FP-478 & GATAACTCAGCCTYTGTTGCGT & $56.4^{\circ} \mathrm{C}$ \\
\hline M. spicatum FP-478 & GATAACTCAGCCTITGTTGCGC & $56.9^{\circ} \mathrm{C}$ \\
\hline Universal RP478 & ATGCTGAGCTCCAAGGGGCA & $61.8^{\circ} \mathrm{C}$ \\
\hline 5' FAM TAG (M. sibiricum) & GAAGGTGACCAAGTTCATGCT & \\
\hline 5' HEX TAG (M. spicatum) & GAAGGTCGGAGTCAACGGATT & \\
\hline
\end{tabular}

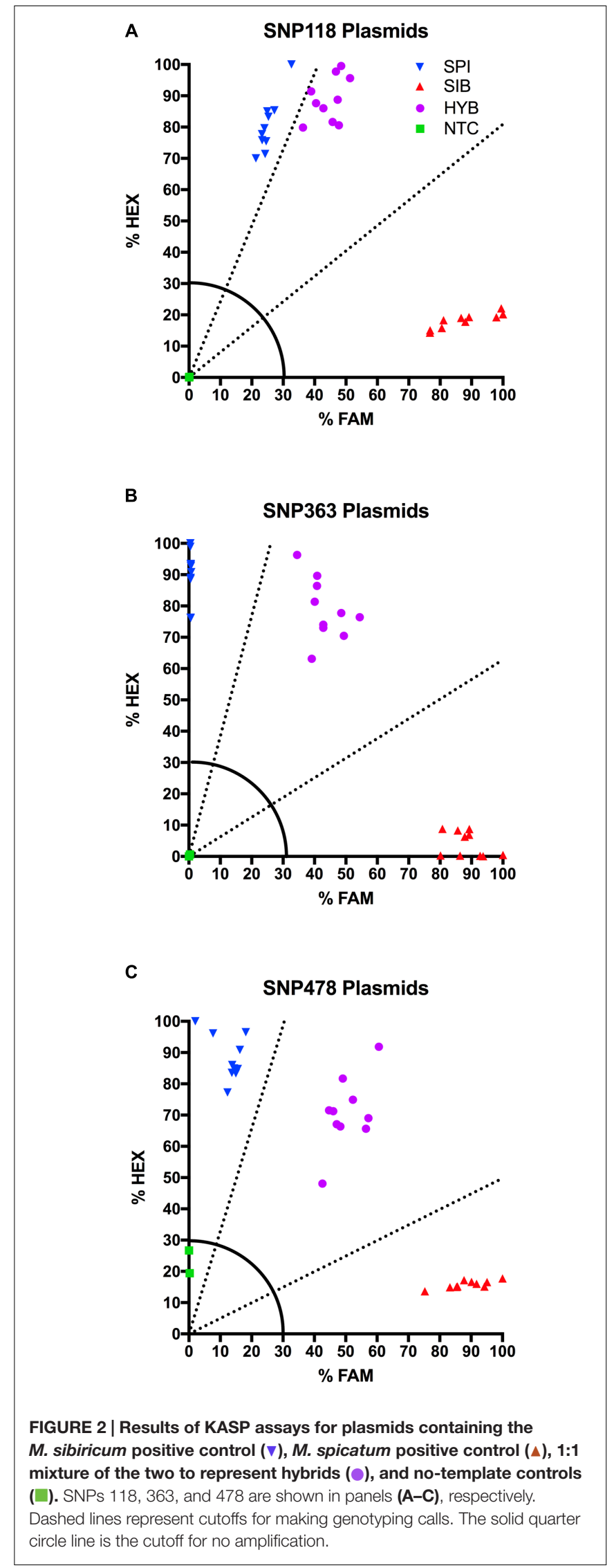


TABLE 3 | Kompetitive Allele Specific PCR SNP genotyping calls and probability of accuracy (Prob) for eight known M. spicatum (M. spi, dark gray) biotypes and eight known hybrid (Hyb, light gray) watermilfoil (M. spicatum $\times$ M. sibiricum) biotypes.

\begin{tabular}{|c|c|c|c|c|c|c|c|c|}
\hline \multirow[t]{2}{*}{ Sample } & \multicolumn{2}{|c|}{ All three SNPs } & \multicolumn{2}{|c|}{ SNP 118} & \multicolumn{2}{|c|}{ SNP 363} & \multicolumn{2}{|c|}{ SNP 478} \\
\hline & Call & Prob & Call & Prob & Call & Prob & Call & Prob \\
\hline Norway & M. spi & 1.00 & M. spi & 0.76 & M. spi & 1.00 & M. spi & 1.00 \\
\hline Hayden & Hyb & 1.00 & Hyb & 1.00 & Hyb & 1.00 & Hyb & 1.00 \\
\hline Mattoon & Hyb & 1.00 & Hyb & 1.00 & Hyb & 1.00 & Hyb & 1.00 \\
\hline Houghton & Hyb & 1.00 & Hyb & 1.00 & Hyb & 1.00 & Hyb & 1.00 \\
\hline CSU KCK & M. spi & 1.00 & M. spi & 0.99 & M. spi & 1.00 & M. spi & 1.00 \\
\hline Alpine 2 & Hyb & 1.00 & Hyb & 1.00 & Hyb & 1.00 & Hyb & 1.00 \\
\hline Alpine 3 & Hyb & 1.00 & Hyb & 1.00 & Hyb & 1.00 & Hyb & 1.00 \\
\hline Richard Farm & Hyb & 1.00 & Hyb & 1.00 & Hyb & 1.00 & Hyb & 1.00 \\
\hline $4 \mathrm{BC}$ & M. spi & 1.00 & M. spi & 0.95 & M. spi & 1.00 & M. spi & 1.00 \\
\hline St Helens & M. spi & 1.00 & M. spi & 0.89 & M. spi & 1.00 & M. spi & 1.00 \\
\hline Jeff & Hyb & 1.00 & Hyb & 1.00 & Hyb & 1.00 & Hyb & 1.00 \\
\hline Hall & M. spi & 1.00 & M. spi & 0.95 & M. spi & 1.00 & M. spi & 0.99 \\
\hline Stoney 2 & M. spi & 1.00 & M. spi & 0.78 & M. spi & 1.00 & M. spi & 0.98 \\
\hline Fawn & M. spi & 1.00 & M. spi & 0.95 & M. spi & 1.00 & M. spi & 1.00 \\
\hline Alpine 1 & Hyb & 1.00 & Hyb & 1.00 & Hyb & 1.00 & Hyb & 1.00 \\
\hline Hanbury & M. spi & 1.00 & M. spi & 0.99 & M. spi & 1.00 & M. spi & 1.00 \\
\hline
\end{tabular}

multiple SNP genotyping assays can be integrated into a single model, increasing the robustness of species diagnostics.

Here we describe KASP assays for three SNPs in the ITS region to genotype individuals from both parental watermilfoil species and their hybrid, using synthesized plasmids containing the appropriate sequences as positive controls. Using these three KASP assays, we genotyped 23 and 16 individuals, respectively, from two lakes, giving a highly accurate picture of Myriophyllum species distribution dynamics in each case. Discriminant analysis showed that while a single SNP was generally sufficient for genotyping an individual, using multiple SNPs increased the reliability of genotyping.

\section{MATERIALS AND METHODS}

\section{Plant Collection}

Several previously identified M. spicatum biotypes and known inter-specific watermilfoil hybrid (M. spicatum $\times$ M. sibiricum) biotypes (eight biotypes each) were harvested from aquaponics cultures maintained in the CSU Weed Research lab. Unknown Myriophyllum individuals were collected from the following two lakes in northern Colorado: Rainbow Lake located at 40.506758, -104.989224 , and Walleye Lake at 40.505680, -104.982883. Individual stems (Rainbow, $n=23$; Walleye, $n=16$ ) were collected from each lake by rake throws. A single leaf from a stem was used for extraction and therefore a tissue sample is expected to represent a unique individual. Tissue samples were stored in sealed bags with damp paper towels at $4^{\circ} \mathrm{C}$ until DNA extraction.

\section{Plant DNA Extraction}

DNA was extracted from $50 \mathrm{mg}$ of watermilfoil leaf tissue using a modified CTAB method (Doyle, 1991). All steps were performed at room temperature $\left(22^{\circ} \mathrm{C}\right)$ unless otherwise indicated and all chemicals were of molecular biology grade. In brief, tissue was initially ground to a fine powder with a metal bead in $500 \mu \mathrm{L}$ of $2 \mathrm{x}$ CTAB buffer (2\% CTAB, $1 \%$ PVP, TRIS-EDTA $\mathrm{pH} 5$ ) using a TissueLyser II (Qiagen, Germantown, MD, USA) at 30 oscillations/second for $1 \mathrm{~min}$. Ground samples were incubated at $65^{\circ} \mathrm{C}$ for $1 \mathrm{~h}$, after which $500 \mu \mathrm{L}$ of phenol:chloroform:isoamyl alcohol (25:24:1) was added. The samples were slowly rocked on an orbital shaker for $15 \mathrm{~min}$. Samples were centrifuged at $10,000 \times g$ for $5 \mathrm{~min}$. The upper phase was transferred to a new tube, to which $500 \mu \mathrm{L}$ of chloroform:isoamyl alcohol (24:1) was added. The samples were again centrifuged at $10,000 \times g$ for $5 \mathrm{~min}$. The upper phase was transferred to a new tube and nucleic acids were precipitated using 0.1 volumes of $3 \mathrm{M}$ sodium acetate and 2.5 volumes of $100 \%$ ethanol. Samples were precipitated at $4^{\circ} \mathrm{C}$ for $15 \mathrm{~min}$ and then centrifuged at $15,000 \times g$ for $15 \mathrm{~min}$. The resulting pellets were re-suspended in $50 \mu \mathrm{L}$ of sterilized water. DNA concentrations and quality were assessed using a spectrophotometer (NanoDrop 2000 Spectrophotometer, Thermo Fisher Scientific, Wilmington, DE, USA). Samples were subsequently diluted to $5 \mathrm{ng} / \mu \mathrm{L}$ for use in all KASP assays.

\section{Plasmid Design}

Two plasmids were designed as positive controls for the KASP assay. Plasmid inserts consisted of the sequence within the ITS region complementary to the genotyping primers, with all interprimer sequence removed (Figure 1 and Table 1). The complete oligonucleotides were synthesized by GenScript (Piscataway, NJ, USA) in the pUC57-Kan plasmid.

Control plasmids were transformed into $\mathrm{DH} 5 \alpha$ E. coli cells using a standard heat transformation protocol (provided by GenScript) and selection on LB plates using $50 \mu \mathrm{g} / \mathrm{mL}$ kanamycin. Individual colonies were transferred to a numbered patch plate and allowed to grow at $37^{\circ} \mathrm{C}$ for $16 \mathrm{~h}$. 
A

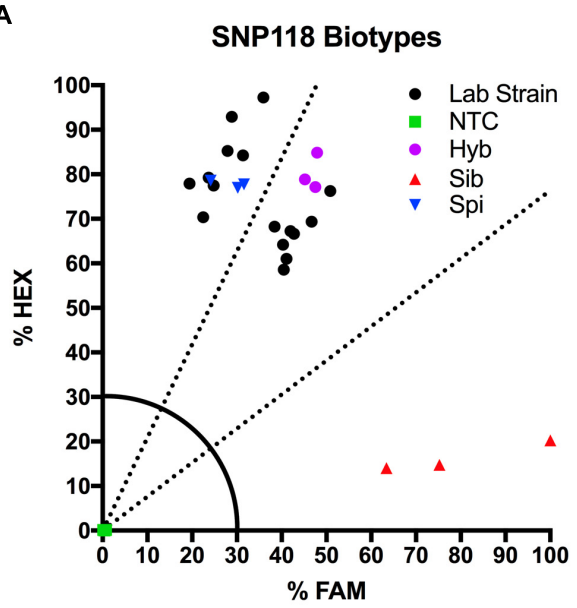

B

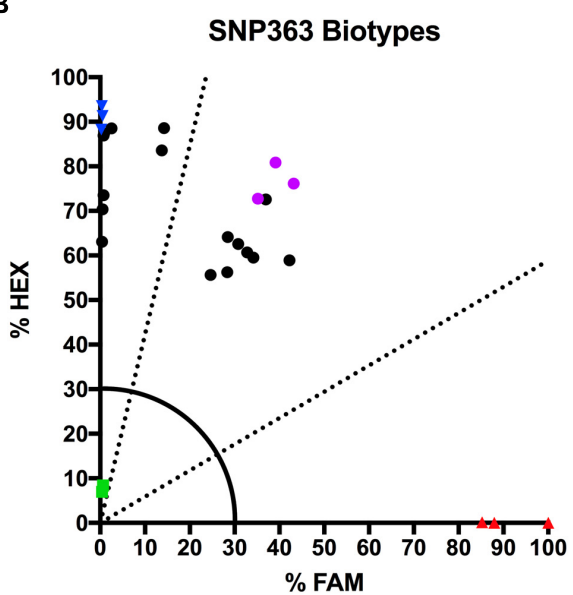

C
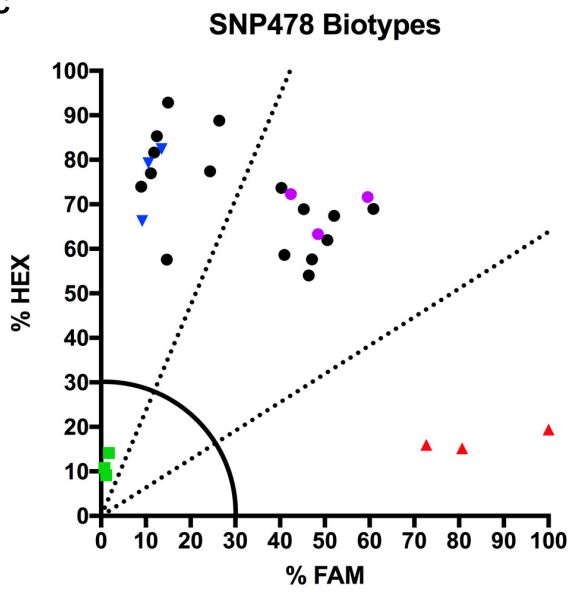

FIGURE 3 | Results of KASP assays for SNPs 118 (A), 363 (B), and 478 (C) from 16 lab biotypes (eight known inter-specific hybrids and eight known M. spicatum biotypes; $\mathbf{0}$ ), as well as the $M$. sibiricum positive control ( $\mathbf{v})$, M. spicatum positive control $(\boldsymbol{\Lambda})$, a 1:1 mixture of the two to represent hybrids (๑), and no-template controls ( $\square$ ). Dashed lines represent cutoffs for making genotyping calls. The solid quarter circle line is the cutoff for no amplification.

\section{E. coli DNA Extraction}

DNA was extracted from cultures grown from ten colonies on each patch plate. A toothpick was dipped into the E. coli colony and used to inoculate $1 \mathrm{~mL}$ of LB containing $50 \mu \mathrm{g} / \mathrm{mL}$ kanamycin. After incubating for $16 \mathrm{~h}$ at $37^{\circ} \mathrm{C}$ with shaking, the E. coli cultures were pelleted by centrifugation at $8,000 \times g$. DNA was extracted from the pellets using Qiaprep Spin Miniprep kit (Qiagen, Germantown, MD, USA) according to the manufacturer's recommendations. DNA concentrations and quality were assessed using a NanoDrop 2000 spectrophotometer. Extracted plasmids were subsequently diluted to $5 \mathrm{pg} / \mu \mathrm{L}$ for use in all KASP assays. A 1:1 mixture of the diluted plasmids was used in KASP assays to simulate an inter-specific hybrid.

\section{Primer Design}

Three primer sets were designed for the KASP assay to distinguish three diagnostic SNPs at bp 118, 363, and 478 in the Internally Transcribed Spacer (ITS) region (Moody et al., 2016; Table 2). For each primer set, the forward primer for M. spicatum was appended at the $5^{\prime}$ end with the sequence complementary to the HEX fluorophore quencher, while the forward primer for $M$. sibiricum was appended at the $5^{\prime}$ end with the sequence complementary to the FAM fluorophore quencher. The forward primers for M. sibiricum at SNPs 118 and 478 spanned sequences containing SNPs that discriminate between sub-populations, which required the use of degenerate bases in the primers. Degenerate bases are indicated according to the universal IUPAC code (Cornish-Bowden, 1985). The forward primers for SNPs 118 and 478 in M. sibiricum designed to amplify these degenerate bases were an equal blend of the two possible alleles at the degenerate SNP.

\section{KASP Assay}

A primer master mix including forward and reverse primers for a single SNP was made according to the KASP assay manufacturer's recommendations (LGC Genomics, Beverly, MA, USA). After all primers were re-suspended in Tris- $\mathrm{HCl}, \mathrm{pH} 8.3$, at $100 \mu \mathrm{M}$, a primer master mix was assembled with $18 \mu \mathrm{L}$ of the M. spicatum forward primer, $18 \mu \mathrm{L}$ of the M. sibiricum forward primer, $45 \mu \mathrm{L}$ of the common reverse primer, and $69 \mu \mathrm{L}$ of $10 \mathrm{mM}$ Tris- $\mathrm{HCl}$, $\mathrm{pH}$ 8.3. KASP master mixes were made for each SNP assay with $432 \mu \mathrm{L}$ LGC Genomics Master Mix (which includes polymerase, dNTPs, buffer, and HEX- and FAM-tagged oligonucleotides) and $11.88 \mu \mathrm{L}$ of the appropriate primer master mix.

Kompetitive Allele Specific PCR reactions were assembled in a 96-well plate with $4 \mu \mathrm{L}$ of master mix and either $4 \mu \mathrm{L}$ water (no-template control), $4 \mu \mathrm{L}$ genomic DNA at $5 \mathrm{ng} / \mu \mathrm{L}$, or $4 \mu \mathrm{L}$ of plasmid DNA at $5 \mathrm{pg} / \mu \mathrm{L}$. Reactions were performed in a BioRad CFX Connect (Bio-Rad Laboratories, Inc., Hercules, CA, USA) according to the following standard KASP PCR program: activation at $94^{\circ} \mathrm{C}$ for $15 \mathrm{~min}$, then 10 touchdown cycles of $94^{\circ} \mathrm{C}$ for $20 \mathrm{~s}$ (denaturing), $61-55^{\circ} \mathrm{C}$ for $60 \mathrm{~s}$ (dropping $0.6^{\circ} \mathrm{C}$ per cycle, for annealing and elongation), $23^{\circ} \mathrm{C}$ for $30 \mathrm{~s}$ (to permit accurate plate reading), followed by 26 cycles of $94^{\circ} \mathrm{C}$ for $20 \mathrm{~s}$, $55^{\circ} \mathrm{C}$ for $60 \mathrm{~s}, 23^{\circ} \mathrm{C}$ for $30 \mathrm{~s}$. Fluorescence was tracked in realtime with plate reads at the end of every amplification cycle. 
Fluorescence data from the cycle showing the greatest distinction between clusters without any background amplification was used for genotyping, which was determined to be cycles $22-24$ of the amplification phase.

\section{Data Analysis}

Due to slight variations in maximum fluorescence and fluorescence in the no-template controls between plates, HEX and FAM fluorescence for each data point were transformed as a percentage of the maximum fluorescence for each fluorophore within a plate. Maximum fluorescence is defined as the highest FAM or HEX signal from any reaction in a 96-well plate. Cutoffs for genotyping calls on unknown samples were drawn by calculating the point halfway between the mean $(x, y)$ coordinate of the control (plasmid) hybrid and either the control M. sibiricum or M. spicatum clusters, then drawing a line from that point to the origin $(0,0)$. Additionally, a zone of "no amplification" was defined as $30 \%$ based on the maximum fluorescence observed in no-template controls. The $30 \%$ cut-off was selected because technical variation at low fluorescence values is more likely to influence genotyping calls than at high fluorescence values, and based on the observation that none of our no-template controls exceeded $30 \%$. A quarter circle at $30 \%$ around the axis intercept was used to define this zone. Genotypes were assigned to unknown samples based on the sector of the plot where their fluorescence values occurred. If a sample fell within the bounds of a zone it was assigned that genotyping call.

Once each sample (experimental samples as well as plasmid controls) was assigned a genotype based on its zone, linear discriminant analysis was performed in JMP 12.2 (SAS Institute Inc., Cary, NC, USA) to evaluate the probability of that individual having its assigned genotype. Genotyping results from each SNP were first assessed independently, then using all three SNPs combined to provide more robust probabilities.

\section{RESULTS}

We developed three KASP primer sets that distinguish between the native $M$. sibiricum and the invasive $M$. spicatum species as well as inter-specific hybrids. Our KASP primers utilize the previously identified SNPs at base pairs 118, 363, and 478 of the ITS region (Table 2). We tested the primer sets on plasmids containing known sequences, on known lab biotypes of M. spicatum and hybrids, and on unknown Myriophyllum individuals harvested from two lakes in northern Colorado. We assigned genotypes manually, and then measured the reliability of the genotyping calls using discriminant analysis to assign probabilities to calls from each SNP individually as well as using all three SNPs together.

TABLE 4 | Kompetitive Allele Specific PCR SNP genotyping calls and probability of accuracy (Prob) for 23 unknown watermilfoil individuals from Rainbow Lake. M. spi (dark gray) = M. spicatum.

\begin{tabular}{|c|c|c|c|c|c|c|c|c|}
\hline \multirow[t]{2}{*}{ Sample } & \multicolumn{2}{|c|}{ All three SNPs } & \multicolumn{2}{|c|}{ SNP 118} & \multicolumn{2}{|c|}{ SNP 363} & \multicolumn{2}{|c|}{ SNP 478} \\
\hline & Call & Prob & Call & Prob & Call & Prob & Call & Prob \\
\hline Plant 1 & M. spi & 1.00 & M. spi & 1.00 & M. spi & 1.00 & M. spi & 1.00 \\
\hline Plant 2 & M. spi & 1.00 & M. spi & 1.00 & M. spi & 1.00 & M. spi & 1.00 \\
\hline Plant 3 & M. spi & 1.00 & M. spi & 1.00 & M. spi & 1.00 & M. spi & 1.00 \\
\hline Plant 4 & M. spi & 1.00 & M. spi & 1.00 & M. spi & 1.00 & M. spi & 1.00 \\
\hline Plant 5 & M. spi & 1.00 & M. spi & 1.00 & M. spi & 1.00 & M. spi & 1.00 \\
\hline Plant 6 & M. spi & 1.00 & M. spi & 1.00 & M. spi & 1.00 & M. spi & 1.00 \\
\hline Plant 7 & M. spi & 1.00 & M. spi & 1.00 & M. spi & 1.00 & M. spi & 1.00 \\
\hline Plant 8 & M. spi & 1.00 & M. spi & 1.00 & M. spi & 1.00 & M. spi & 1.00 \\
\hline Plant 9 & M. spi & 1.00 & M. spi & 1.00 & M. spi & 1.00 & M. spi & 1.00 \\
\hline Plant 10 & M. spi & 1.00 & M. spi & 1.00 & M. spi & 1.00 & M. spi & 1.00 \\
\hline Plant 11 & M. spi & 1.00 & M. spi & 1.00 & M. spi & 1.00 & M. spi & 1.00 \\
\hline Plant 12 & M. spi & 1.00 & M. spi & 1.00 & M. spi & 1.00 & M. spi & 1.00 \\
\hline Plant 13 & M. spi & 1.00 & M. spi & 1.00 & M. spi & 1.00 & M. spi & 1.00 \\
\hline Plant 14 & M. spi & 1.00 & M. spi & 1.00 & M. spi & 1.00 & M. spi & 1.00 \\
\hline Plant 15 & M. spi & 1.00 & M. spi & 1.00 & M. spi & 1.00 & M. spi & 1.00 \\
\hline Plant 16 & M. spi & 1.00 & M. spi & 1.00 & M. spi & 1.00 & M. spi & 1.00 \\
\hline Plant 17 & M. spi & 1.00 & M. spi & 0.98 & M. spi & 1.00 & M. spi & 1.00 \\
\hline Plant 18 & M. spi & 1.00 & M. spi & 1.00 & M. spi & 1.00 & M. spi & 1.00 \\
\hline Plant 19 & M. spi & 1.00 & M. spi & 1.00 & M. spi & 1.00 & M. spi & 1.00 \\
\hline Plant 20 & M. spi & 1.00 & M. spi & 0.88 & M. spi & 1.00 & M. spi & 1.00 \\
\hline Plant 21 & M. spi & 1.00 & M. spi & 1.00 & M. spi & 1.00 & M. spi & 1.00 \\
\hline Plant 22 & M. spi & 1.00 & M. spi & 1.00 & M. spi & 1.00 & M. spi & 1.00 \\
\hline Plant 23 & M. spi & 1.00 & M. spi & 0.085 & M. spi & 1.00 & M. spi & 1.00 \\
\hline
\end{tabular}

Bolded font indicates that the genotyping call made by the point's location does not agree with the linear discriminant analysis. 

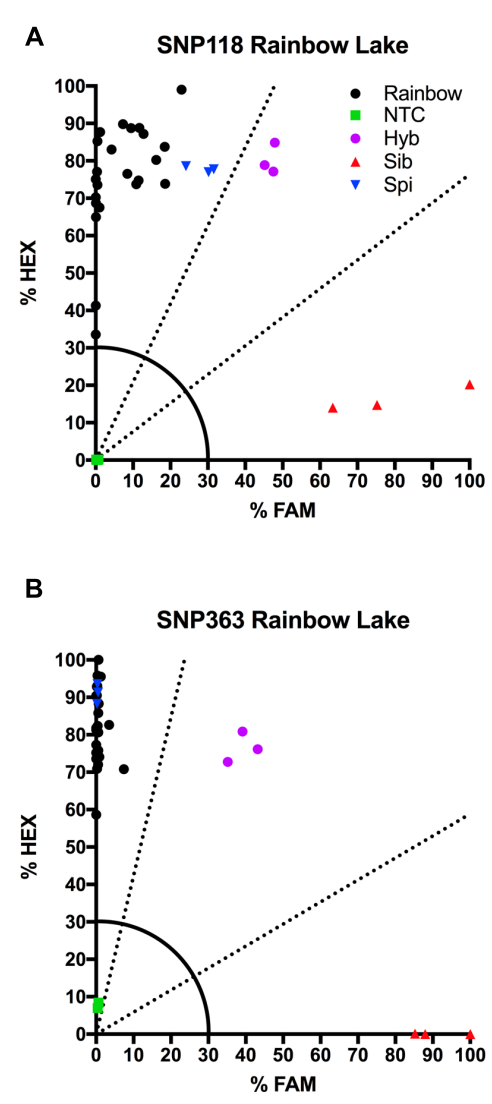

C

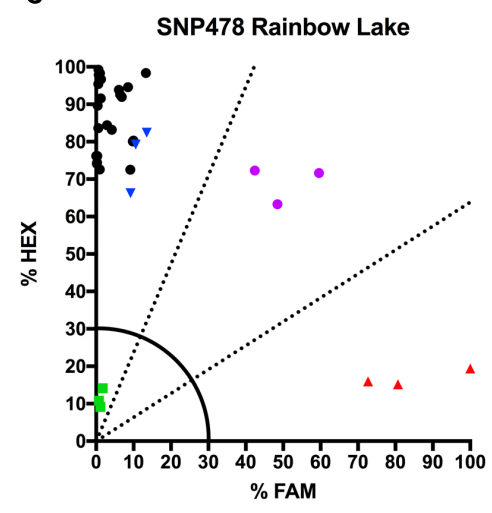

D

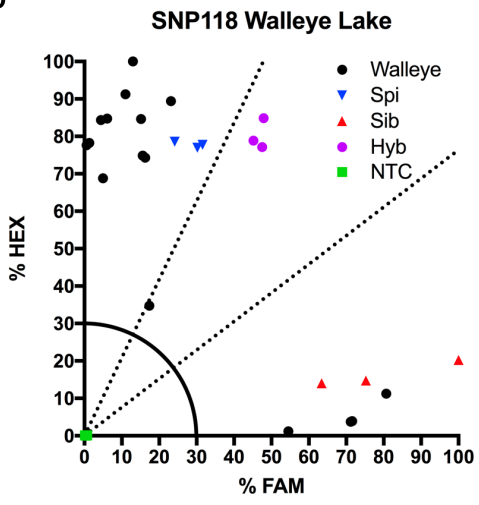

E

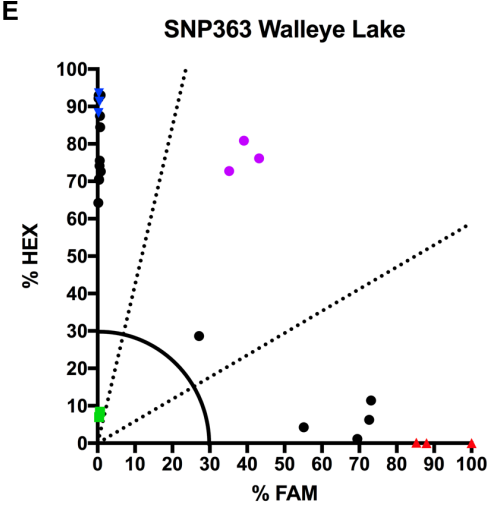

F SNP478 Walleye Lake

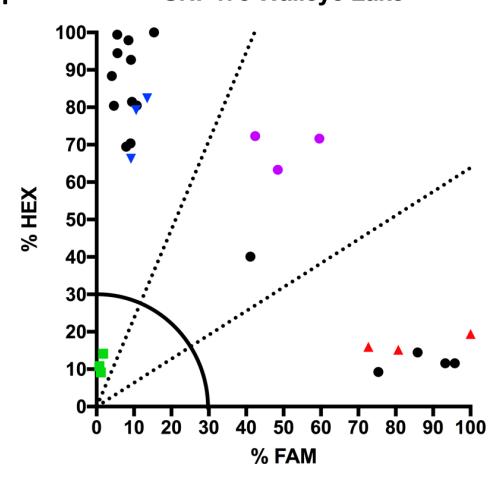

FIGURE 4 | Results of KASP assays from wild collections of unknown watermilfoil individuals from Rainbow Lake for SNP 118 (A), SNP 363 (B), and SNP 478 (C), and from Walleye Lake for SNP 118 (D), SNP 363 (E), and SNP 478 (F). Samples are represented as follows: M. sibiricum positive control ( $\mathbf{V})$,

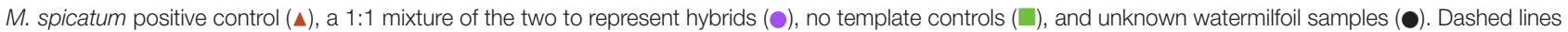
represent cutoffs for making genotyping calls. The solid quarter circle line is the cutoff for no amplification.

\section{KASP Assays on Plasmids}

We developed two plasmids (Figure 1) to serve as positive controls for the KASP-PCR. Plasmid controls were ideal because they allow for rapid generation of DNA of a known genotype and eliminate the need to maintain both species of Myriophyllum as well as the inter-specific hybrid in hydroponic culture as positive genotyping controls.
The plasmid DNA performed consistently from assay to assay and allowed us to more accurately characterize unknown individuals in the KASP assay. For SNP 118, SNP 363, and SNP 478, we tested ten distinct E. coli colonies. All ten samples containing a given genotype formed a tight, distinct cluster on the HEX-FAM $x-y$ plot with fluorescence values well above the $30 \%$ cut-off (Figure 2). SNP 118 had a very clear M. sibiricum 
cluster, but the M. spicatum and the 1:1 synthetic hybrids were relatively close to each other, due to increased FAM fluorescence for the M. spicatum samples (Figure 2A). However, there was no overlap between the M. spicatum and the synthetic hybrid sample clusters. SNP 363 and SNP 478 show obvious separation of the fluorescence signal from each of the three possible genotypes, with the $M$. spicatum plasmids having almost exclusively HEX signal, $M$. sibiricum plasmids having almost exclusively FAM signal, and the 1:1 mixture of each genotype having both HEX and FAM signals (Figures 2B,C). No plasmid had an ambiguous call or fell below the $30 \%$ fluorescence threshold for any of the three SNPs. This test confirmed the utility of plasmids as internal positive controls for plant genotyping assays.

\section{KASP Assays on Lab Biotypes}

We tested several biotypes of Myriophyllum that are maintained in aquaponics culture at CSU. These biotypes were originally collected from various locations in North America (Table 3). The KASP results from all three SNP primer sets showed that eight of these biotypes clustered with the M. spicatum plasmid control, with high HEX signal and minimal FAM signal (Norway, CSU KCK, 4BC, St Helens, Hall, Stoney 2, Fawn, Hanbury), while eight clustered with the 1:1 synthetic hybrid mixture of $M$. spicatum and $M$. sibiricum plasmid controls, with approximately equal HEX and FAM fluorescent signals (Hayden, Mattoon, Houghton, Alpine 2, Alpine 3, Richard Farm, Jeff, Alpine 1) (Table 3 and Figure 3). The genotyping calls from the KASP assay matched the known genotypes of the samples exactly.

The probability that a genotype call was correct was calculated by performing discriminant analysis on the corrected fluorescence data for each SNP separately and for all three SNPs together (Table 3). Particularly for SNP118, several individuals had a reduced probability that the genotype was correct (e.g., Norway or Stoney 2). However, when all three SNPs were considered together, the probability was $100 \%$ for each genotype call (Table 3). These results confirm that all three SNPs are strongly linked and co-inherited and therefore the three SNPs can be used together to provide accurate genotyping, as would be expected for SNPs in the ITS2 region.

\section{KASP Assays on Rainbow and Walleye Lake}

We also tested our assay on wild, unknown individuals from two lakes in northern Colorado, Rainbow Lake $(n=23)$ and Walleye Lake $(n=16)$. For Rainbow Lake, all sampled individuals were identified as the invasive $M$. spicatum, as the fluorescence signal from all three SNPs for each individual was predominantly the HEX wavelength (Table 4 and Figures 4A-C). Only the genotyping call of $M$. spicatum for plant 23 for SNP 118 was unsupported by the linear discrimination analysis $(P=0.085)$. The analysis assigned this call as "No amplification" with $P=0.856$. However, when all three SNPs were considered together, a call of $M$. spicatum for Plant 23 was predicted with $P=1.0$. Samples from Walleye Lake were identified as M. spicatum, M. sibiricum and inter-species hybrid genotypes, with 11 individuals showing predominantly HEX fluorescence and clustering with the M. spicatum plasmid controls, while four individuals (plants 2, 3, 8, and 12) showed predominantly FAM fluorescence and clustered with the M. sibiricum plasmid controls (Table 5 and Figures 4D-F). Additionally, one individual (plant 1) was identified as the hybrid genotype, as all three SNPs showed unambiguous dual HEX and FAM fluorescence and clustered with the artificial hybrid (Table 5 and Figures 4D-F).

TABLE 5 | Kompetitive Allele Specific PCR SNP genotyping calls and probability of accuracy (Prob) for 16 unknown watermilfoil individuals from Walleye Lake.

\begin{tabular}{|c|c|c|c|c|c|c|c|c|}
\hline \multirow[t]{2}{*}{ Sample } & \multicolumn{2}{|c|}{ All three SNPs } & \multicolumn{2}{|c|}{ SNP 118} & \multicolumn{2}{|c|}{ SNP 363} & \multicolumn{2}{|c|}{ SNP 478} \\
\hline & Call & Prob & Call & Prob & Call & Prob & Call & Prob \\
\hline Plant 1 & Hyb & 1.00 & Hyb & 0.49 & Hyb & 1.00 & Hyb & 1.00 \\
\hline Plant 2 & M. sib & 1.00 & M. sib & 1.00 & M. sib & 1.00 & M. sib & 1.00 \\
\hline Plant 3 & M. sib & 1.00 & M. sib & 1.00 & M. sib & 1.00 & M. sib & 1.00 \\
\hline Plant 4 & M. spi & 1.00 & M. spi & 1.00 & M. spi & 1.00 & M. spi & 1.00 \\
\hline Plant 5 & M. spi & 1.00 & M. spi & 1.00 & M. spi & 1.00 & M. spi & 1.00 \\
\hline Plant 6 & M. spi & 1.00 & M. spi & 1.00 & M. spi & 1.00 & M. spi & 1.00 \\
\hline Plant 7 & M. spi & 1.00 & M. spi & 1.00 & M. spi & 1.00 & M. spi & 1.00 \\
\hline Plant 8 & M. sib & 1.00 & M. sib & 1.00 & M. sib & 1.00 & M. sib & 1.00 \\
\hline Plant 9 & M. spi & 1.00 & M. spi & 1.00 & M. spi & 1.00 & M. spi & 1.00 \\
\hline Plant 10 & M. spi & 1.00 & M. spi & 1.00 & M. spi & 1.00 & M. spi & 1.00 \\
\hline Plant 11 & M. spi & 1.00 & M. spi & 0.99 & M. spi & 1.00 & M. spi & 1.00 \\
\hline Plant 12 & M. sib & 1.00 & M. sib & 1.00 & M. sib & 1.00 & M. sib & 1.00 \\
\hline Plant 13 & M. spi & 1.00 & M. spi & 1.00 & M. spi & 1.00 & M. spi & 1.00 \\
\hline Plant 14 & M. spi & 1.00 & M. spi & 1.00 & M. spi & 1.00 & M. spi & 1.00 \\
\hline Plant 15 & M. spi & 1.00 & M. spi & 1.00 & M. spi & 1.00 & M. spi & 1.00 \\
\hline Plant 16 & M. spi & 1.00 & M. spi & 0.99 & M. spi & 1.00 & M. spi & 1.00 \\
\hline
\end{tabular}

M. spi (dark gray) = M. spicatum; Hyb (light gray) = inter-specific hybrid (M. spicatum $\times$ M. sibiricum); M. sib (white) = M. sibiricum. 


\section{DISCUSSION}

Discriminant analysis verified the accuracy of the genotyping calls, with SNP 118 producing a few lower-confidence genotype calls (for plants 20 and 23 from Rainbow Lake and plant 1 from Walleye Lake) but $100 \%$ probability of a correct call when data from all three SNPs were considered simultaneously. The forward primers for both SNP 118 and SNP 478 used one degenerate base each; however, the calls for SNP 478 appear to be much more accurate than for SNP 118. The degenerate bases in each case were required to allow efficient amplification of ITS sequences, because SNPs exist in the forward primers that distinguish between different sub-populations of $M$. sibiricum, but not between $M$. spicatum and $M$. sibiricum. Performance differences in the three KASP markers may be attributed to the type and location of the degenerate base. For the SNP118 forward primer, a $\mathrm{W}$ indicates an $\mathrm{A}$ or $\mathrm{T}$ which is either a purine or pyrimidine, respectively, while the forward primer for SNP478 contains a Y indicating a C or T, which are both pyrimidines. Degenerate bases may lower optimal annealing temperature of the primer, which in turn lowers the ability of the primer set to distinguish between our two species. The use of multiple SNPs together for genotype identification overcomes inefficiencies of any one marker, as they are always co-inherited.

The ability to genotype dozens of individuals provides the ability to identify the presence of rare individuals, such as a less common parental species or the inter-specific hybrid. Lakes with complex species distribution dynamics, such as low proportion of hybrids, are where herbicide application must be carefully chosen so as not to select for the more vigorous and less herbicide-sensitive hybrid individuals. Only recently were hybrids suspected (Moody and Les, 2002), and then determined to be more invasive and less herbicide sensitive (LaRue et al., 2013). The exact distribution, population sizes, and proportions of hybrid, $M$. sibiricum, and M. spicatum are largely unknown, in part due to the cost and limited throughput of current genotyping methods. The genetic diversity of the two Myriophyllum species in the ITS region has been explored by Sturtevant et al. (2009), including characterization of the various within-species ITS genotypes that exist. Our KASP markers are useful to distinguish between $M$. spicatum, M. sibiricum and their interspecific hybrid, but not to distinguish within-species genetic variation.

\section{REFERENCES}

Berger, S. T., Netherland, M. D., and MacDonald, G. E. (2015). Laboratory documentation of multiple-herbicide tolerance to fluridone, norflurazon, and topramazone in a hybrid watermilfoil (Myriophyllum spicatum $\times$ M. sibiricum) population. Weed Sci. 63, 235-241. doi: 10.1614/WS-D-1400085.1

Coffey, B. T., and McNabb, C. D. (1974). Eurasian water-milfoil in Michigan. Mich. Bot. 13, 159-165.

Cornish-Bowden, A. (1985). Nomenclature for incompletely specified bases in nucleic acid sequences: recommendations 1984. Nucleic Acids Res. 13, 3021-3030. doi: 10.1093/nar/13.9.3021

Couch, R., and Nelson, E. (1988). Myriophyllum quitense (Haloragaceae) in the United States. Brittonia 40, 85-88. doi: 10.2307/2806880
Monitoring has been conducted in some areas of North America (e.g., Zuellig and Thum, 2012; Grafe et al., 2015; Moody et al., 2016), but the distribution of the invasive species on the continental scale remains undetermined. With the ability to genotype hundreds of individuals rapidly and inexpensively using KASP, aquatic weed managers will be able to make more informed decisions about herbicide type and application rates, such as choosing specific herbicides and rates to control rare hybrid individuals only when they are confirmed to be present. Appropriate sampling structures would be critical, including spatially dispersed locations within a lake, to avoid redundant sampling of any clonal plants and ensuring detection of potentially rare hybrid individuals. Larger data sets comprised of accurate genotyping data will allow modeling of Myriophyllum species distribution dynamics, testing the hypothesis that increased selection pressure from herbicide application favors hybrid individuals due to their decreased herbicide sensitivity. Lakes can be genotyped using KASP both before and after herbicide applications to quantify shifts in species distribution dynamics toward invasive M. spicatum or hybrid watermilfoil individuals.

\section{SOURCES OF MATERIALS}

Lake material was collected from two of the lakes at the Swift Ponds at Colorado Youth Outdoors: Rainbow Lake (40.506758, -104.989224) and Walleye Lake (40.505680, - 104.982883).

\section{AUTHOR CONTRIBUTIONS}

EP, MF, KK, SN, and TG designed the experiments; EP, MF, and KK performed the experiments; $\mathrm{EP}, \mathrm{MF}$, and TG analyzed the results; and $\mathrm{EP}, \mathrm{MF}, \mathrm{KK}, \mathrm{SN}$, and TG wrote and approved the manuscript.

\section{FUNDING}

The authors acknowledge United Phosphorus, Inc. (UPI) for contributing funding for this research, and support from the Colorado State University Libraries Open Access Research and Scholarship Fund.

Doyle, J. (1991). "DNA protocols for plants-CTAB total DNA isolation," in 'Molecular Techniques in Taxonomy', eds G. M. Hewitt and A. Johnston (Berlin: Springer), 283-293. doi: 10.1007/978-3-642-83962-7_18

Eiswerth, M. E., Donaldson, S. G., and Johnson, W. S. (2000). Potential environmental impacts and economic damages of Eurasian watermilfoil (Myriophyllum spicatum) in Western Nevada and Northeastern California. Weed Technol. 14, 511-518. doi: 10.1614/0890-037X(2000)014[0511:PEIAED] 2.0.CO;2

Grace, J. B. (1993). The adaptive significance of clonal reproduction in angiosperms: an aquatic perspective. Aquat. Bot. 44, 159-180. doi: 10.1016/ 0304-3770(93)90070-D

Grace, J. B., and Wetzel, R. G. (1978). The production biology of Eurasian watermilfoil (Myriophyllum spicatum L.): a review. J. Aquat. Plant Manage. 16, $1-11$. 
Grafe, S. F., Boutin, C., Pick, F. R., and Bull, R. D. (2015). A PCR-RFLP method to detect hybridization between the invasive Eurasian watermilfoil (Myriophyllum spicatum) and the native northern watermilfoil (Myriophyllum sibiricum), and its application in Ontario lakes. Botany 93, 117-121. doi: 10.1139/cjb-20140135

Hovick, S. M., and Whitney, K. D. (2014). Hybridisation is associated with increased fecundity and size in invasive taxa: meta-analytic support for the hybridisation-invasion hypothesis. Ecol. Lett. 17, 1464-1477. doi: 10.1111/ele. 12355

LaRue, E. A., Zuellig, M. P., Netherland, M. D., Heilman, M. A., and Thum, R. A. (2013). Hybrid watermilfoil lineages are more invasive and less sensitive to a commonly used herbicide than their exotic parent (Eurasian watermilfoil). Evol. Appl. 6, 462-471. doi: 10.1111/eva.12027

Madsen, J. D., Sutherland, J., Bloomfield, J., Eichler, L., and Boylen, C. (1991). The decline of native vegetation under dense Eurasian watermilfoil canopies. J. Aquat. Plant Manage. 29, 94-99.

Moody, M., and Les, D. (2007). Geographic distribution and genotypic composition of invasive hybrid watermilfoil (Myriophyllum spicatum $\times$ M. sibiricum) populations in North America. Biol. Invasions 9, 559-570. doi: 10.1007/s10530-006-9058-9

Moody, M. L., and Les, D. H. (2002). Evidence of hybridity in invasive watermilfoil (Myriophyllum) populations. Proc. Natl. Acad. Sci. U.S.A. 99, 14867-14871. doi: 10.1073/pnas.172391499

Moody, M. L., Palomino, N., Weyl, P. S., Coetzee, J. A., Newman, R. M., Harms, N. E., et al. (2016). Unraveling the biogeographic origins of the Eurasian watermilfoil (Myriophyllum spicatum) invasion in North America. Am. J. Bot. 103, 709-718. doi: 10.3732/ajb.1500476

Olden, J. D., and Tamayo, M. (2014). Incentivizing the public to support invasive species management: Eurasian milfoil reduces lakefront property values. PLoS ONE 9:e110458. doi: 10.1371/journal.pone.0110458
Philbrick, C. T., and Les, D. H. (1996). Evolution of aquatic angiosperm reproductive systems: what is the balance between sexual and asexual reproduction in aquatic angiosperms? Bioscience 46, 813-826. doi: 10.2307/ 1312967

Semagn, K., Babu, R., Hearne, S., and Olsen, M. (2014). Single nucleotide polymorphism genotyping using Kompetitive Allele Specific PCR (KASP): overview of the technology and its application in crop improvement. Mol. Breed. 33, 1-14. doi: 10.1007/s11032-013-9917-x

Sturtevant, A. P., Hatley, N., Pullman, G., Sheick, R., Shorez, D., Bordine, A., et al. (2009). Molecular characterization of Eurasian watermilfoil, northern milfoil, and the invasive interspecific hybrid in Michigan lakes. J. Aquat. Plant Manage. 47, 128-135.

Zuellig, M. P., and Thum, R. A. (2012). Multiple introductions of invasive Eurasian watermilfoil and recurrent hybridization with northern watermilfoil in North America. J. Aquat. Plant Manage. 50, 1-19.

Conflict of Interest Statement: EP, MF, and KK have a patent pending on the genotyping method.

The other authors declare that the research was conducted in the absence of any commercial or financial relationships that could be construed as a potential conflict of interest.

Copyright (C) 2017 Patterson, Fleming, Kessler, Nissen and Gaines. This is an openaccess article distributed under the terms of the Creative Commons Attribution License (CC BY). The use, distribution or reproduction in other forums is permitted, provided the original author(s) or licensor are credited and that the original publication in this journal is cited, in accordance with accepted academic practice. No use, distribution or reproduction is permitted which does not comply with these terms. 